\title{
Contribution of steps to optical properties of vicinal diamond (100):H surfaces
}

\author{
M. Schwitters, ${ }^{1}$ D. S. Martin, ${ }^{1}$ P. Unsworth, ${ }^{1}$ T. Farrell, ${ }^{1}$ J. E. Butler, ${ }^{2}$ M. Marsili, ${ }^{3}$ O. Pulci, ${ }^{3}$ and P. Weightman ${ }^{1}$ \\ ${ }^{1}$ Department of Physics and Surface Science Research Centre, University of Liverpool, L69 7ZE England, United Kingdom \\ ${ }^{2}$ Code 6174, Naval Research Laboratories, Washington, DC 20375, USA \\ ${ }^{3}$ Dipartimento di Fisica, Universit di Roma “Tor Vergata," CNR-INFM-NAST, European Theoretical Spectroscopy Facility (ETSF), \\ Via della Ricerca Scientifica 1, I-00133 Rome, Italy \\ (Received 28 June 2010; revised manuscript received 25 October 2010; published 9 February 2011)
}

\begin{abstract}
We apply reflection anisotropy spectroscopy (RAS) to high-quality atomically smooth $\mathrm{H} / \mathrm{C}(100) 2 \times 1$ surfaces. The optical signal of the $\mathrm{H} / \mathrm{C}(100) 0^{\circ}$ flat surface, and of the $\mathrm{H} / \mathrm{C}(100) 2^{\circ}$ and $\mathrm{H} / \mathrm{C}(100) 4^{\circ}$ vicinal surfaces, is investigated in terms of single and double steps. A comparison of experimental and theoretical results obtained from ab initio calculations shows that, in the energy range considered ( $1-5 \mathrm{eV})$, the RAS response can be interpreted in terms of single height $S_{B}$ and double height $D_{A}$ steps.
\end{abstract}

DOI: 10.1103/PhysRevB.83.085402

PACS number(s): 73.20.At, 78.40.Kc, 78.68.+m

\section{INTRODUCTION}

Diamond is a material with outstanding properties: It is harder than any other material, and it has the highest thermal conductivity. The surface chemical inertness and high degree of biocompatibility make diamond well suited for biomedical applications. Many of the most interesting and potentially useful properties of diamond, such as its negative electron affinity upon hydrogen adsorption ${ }^{1,2}$ and its unique surface conductivity, ${ }^{3,4}$ stem from its surface properties. The significant potential of diamond for technological applications has led to considerable activity in the growth of synthetic materials by processes such as chemical vapor deposition (CVD) ${ }^{5}$ and plasma-assisted chemical vapor deposition (PACVD). ${ }^{6}$ The realization of the potential of diamond in applications such as optoelectronics and electronic devices ${ }^{7,8}$ is dependent not only on controlling the growth of the material but also on understanding its surface electronic structure and in particular the influence of hydrogen. ${ }^{4}$ In order to optimize growth processes it is useful to develop techniques for the in situ monitoring of diamond growth and in particular to monitor the behavior of surface steps, ${ }^{9-14}$ the anisotropic etching of which by atomic hydrogen is known to be important in the production of smooth diamond (100) [C(100)] surfaces. $^{10}$ Reflection anisotropy spectroscopy (RAS) ${ }^{15,16}$ is a technique that has found application in monitoring the growth of $\mathrm{Si}, \mathrm{Ge}$, and III-V compounds ${ }^{17-21}$ and the interfaces that are important in the integration of $\mathrm{Si}, \mathrm{Ge}$, and $\mathrm{GaAs}$ technologies. ${ }^{22-25}$ RAS has been applied to the $\mathrm{C}(100)$ surface $^{26}$ and to the $\mathrm{O} / \mathrm{C}(100)$ surface $^{27}$ and is a suitable technique for monitoring the CVD growth of diamond and in particular surface processes associated with steps. RAS is an optical probe that achieves surface sensitivity by measuring the difference in reflection of normal incidence plane polarized light in two directions at right angles in the surface plane. For a cubic material this leads to a cancellation of the bulk response, and the RAS signal is thus sensitive to anisotropic surface processes. It is well known that surface steps ${ }^{28}$ are important in the growth of $\mathrm{Si}$ and in particular that the control of double height steps through thermal processing of vicinal surfaces can be used to create single-domain $\mathrm{Si}(100)$ surfaces for a variety of applications. ${ }^{19,21,29}$ A combination of experimental and theoretical work ${ }^{19,21,29-35}$ has revealed the RAS signatures of both single (S) and double (D) height steps on $\mathrm{Si}(100),{ }^{30-35}$ and this has proved useful in monitoring the role of steps on this surface.

In this work we report the results of RAS measurements of hydrogen-terminated vicinal C(100) surfaces and establish empirical RAS signatures arising from single and double height steps on these surfaces. We compare the empirical step spectral signatures with the results of theoretical calculations, within Density Functional Theory (DFT) ${ }^{36}$ and the $\mathrm{GW}^{37}$ method, for the flat and stepped surfaces using the models suggested by Chadi ${ }^{28}$ and Tsai and Yeh. ${ }^{14}$

\section{EXPERIMENTAL PROCEDURE}

A type IIb natural diamond of dimensions $8 \times 2.5 \times 4 \mathrm{~mm}^{3}$ and with (100) surfaces was obtained from De Beers. This material was chosen since boron doping, at a level of less than a part per million, makes it conducting, and therefore it is possible to characterize the surface by low-energy electron diffraction (LEED) and x-ray photoelectron spectroscopy (XPS).

A key issue in obtaining control of diamond surfaces for device applications is to produce atomically smooth surfaces. In this work we employed a range of processing techniques at the Naval Research Laboratory, Washington, DC, including mechanical polishing and hydrogen-plasma etching. ${ }^{38}$ The polishing procedure begins with polishing the specimen along its "soft" (100) direction on a high-speed iron scaife impregnated with diamond grit $(6-12 \mu \mathrm{m})$, followed by a second polish with the specimen's "hard" (110) direction aligned toward the scaife motion, using a scaife impregnated with finer grit $(\leqslant 1 \mu \mathrm{m})$. This second step was performed with low load until no polishing grooves were visible using a differential interference contrast reflection microscope with a $5 \times$ objective and $10 \times$ eyepiece. The specimen was cleaned by boiling in aqua regia, then boiling in a $3: 2$ ratio of sulfuric acid : nitric acid, rinsed with deionized water, and stored under ethyl alcohol. A hydrogen plasma treatment was performed in a microwave plasma-enhanced CVD reactor operating at $600 \mathrm{~W}$ in 10 Torr $\mathrm{H}_{2}$ for 30 minutes at a sample temperature of $1100 \mathrm{~K}$. The specimen was then cooled to less than $500 \mathrm{~K}$ in the plasma to maximize the hydrogen termination of the diamond 
surface. In addition to the (100) surface, denoted H/C(100) $0^{\circ}$, vicinal (100) surfaces, orientated $2^{\circ}$ and $4^{\circ}$ toward the [110] direction and denoted $\mathrm{H} / \mathrm{C}(100) 2^{\circ}$ and $\mathrm{H} / \mathrm{C}(100) 4^{\circ}$, were also produced by the polishing-etching procedure and the orientations confirmed by x-ray diffraction.

The specimens were transported to Liverpool under ethyl alcohol, and atomic force microscopy (AFM) experiments were performed on the surfaces produced at various stages of the surface preparation process. Specimens were inserted into the ultrahigh vacuum (UHV) chamber of the Liverpool electron spectrometer ${ }^{39}$ for LEED, XPS, and RAS measurements. Heat treatment of the specimens was carried out in UHV at pressures below $1 \times 10^{-11}$ Torr by electron beam heating of the underside of the specimen, with the temperature during annealing measured with optical pyrometry and with a thermocouple positioned inside a cavity in the specimen via a laser-drilled hole. XPS measurements on the as-received specimens and following various stages of the heat treatment were carried out using monochromated $\mathrm{Al} K \alpha \times$ rays. The XPS results showed that gentle heating to $500 \mathrm{~K}$ in UHV removed adventitious adsorbed hydrocarbons and oxygen species and produced clean $\mathrm{H} / \mathrm{C}(100)$ surfaces with the single-component symmetrical $\mathrm{C}$ 1s photoelectron line shape characteristic of H-terminated C(100) $2 \times 1$ surfaces. $^{40,41}$

RAS measurements were made on the $\mathrm{H}$-terminated $\mathrm{C}(100)$ $0^{\circ}, 2^{\circ}$, and $4^{\circ}$ surfaces in UHV following characterization of the specimens by XPS and LEED. The RAS instrument was of the Aspnes design ${ }^{17}$ and was mounted external to the UHV chamber, projecting and receiving light through a lowstrain window. $\mathrm{RAS}^{15,16}$ measures $\Delta r$, the difference in the normal incidence reflection for light linearly polarized in two orthogonal directions in the surface, normalized to the mean reflection $r$. For $\mathrm{C}(100)$ the RAS is given by

$$
\frac{\Delta r}{r}=\frac{2\left(r_{[110]}-r_{[1 \overline{1} 0]}\right)}{r_{[110]}+r_{[1 \overline{1} 0]}},
$$

where $r$ is the complex Fresnel reflection coefficient. Care was taken to distinguish between reflections from the front and back surfaces of the transparent diamond crystal. The diamond was slightly wedge shaped, and by use of a laser and a reflective Si wafer placed behind the back surface, the two reflections were clearly distinguished. By eliminating the backsurface-reflected beam from the RAS spectrometer, we ensured that only the front surface reflection was measured, and we therefore expect no contributions from signals whose origin are bulk related.

\section{RESULTS}

AFM measurements of the central area of the diamond surface reveal that the surface preparation method leads to an atomically smooth surface. Potential polishing grooves after mechanical polishing have been etched away by the plasma, and the root-mean-square roughness of the surface has been reduced to $\sim 0.1 \mathrm{~nm}$. The surface quality achieved by applying all the preparation steps is demonstrated in UHV by the very sharp $2 \times 1$ LEED pattern of the H/C(100) surface shown in Fig. 1 and by scanning tunneling microscopy (STM) results, which, while of low resolution, show the surface to be smooth, in agreement with the AFM results.

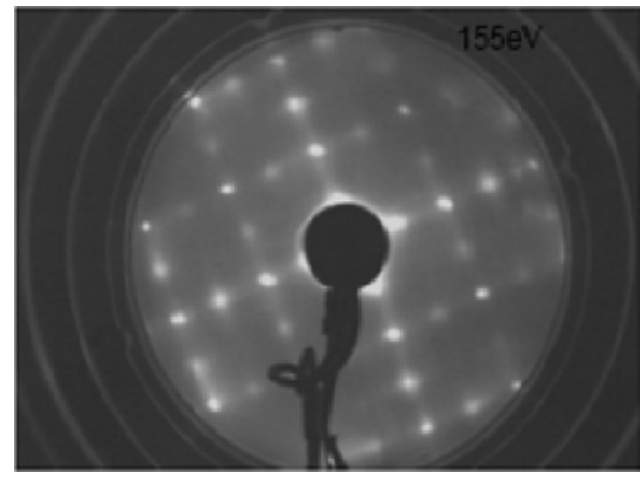

FIG. 1. LEED pattern of the $\mathrm{H} / \mathrm{C}(100) 2^{\circ}$ surface at a beam energy of $155 \mathrm{eV}$ showing the two-domain $(2 \times 1)$ surface reconstruction.

LEED showed the $\mathrm{H} / \mathrm{C}(100) 0^{\circ}, 2^{\circ}$, and $4^{\circ}$ surfaces to have the characteristic $2 \times 1$ pattern expected of the two-domain surfaces. The RAS results for the $\mathrm{H} / \mathrm{C}(100) 0^{\circ}, 2^{\circ}$, and $4^{\circ}$ $2 \times 1$ surfaces are shown in Fig. 2. Figure 3(a) shows in detail the RAS profile of the $\mathrm{H} / \mathrm{C}(100) 0^{\circ}$ surface. It should be noted that the results shown in Fig. 2 were obtained at different times following the progressive polishing of the specimen to increase the vicinality. It is thus difficult to be sure that accurate corrections have been made for systematic errors, particularly in accurately reproducing the zero of the RAS signal in different experiments since this is sensitive to minutes of arc in the angle of the first polariser of the RAS instrument. A recent study ${ }^{42}$ showed that this lack of reproducibility of the zero RAS signal could be as high as $0.25 \times 10^{-3}$.

\section{DISCUSSION}

\section{A. Surface geometry}

Our AFM, STM, XPS, and LEED results show that the polishing process developed in the Naval Research Laboratory is able to produce high-quality atomically smooth H-terminated C(100) surfaces. We now consider the geometry of the surfaces produced by this process. The LEED pattern of the $\mathrm{H} / \mathrm{C}(100)$ $0^{\circ}$ surface showed half-order spots from $2 \times 1$ and $1 \times 2$ terraces of roughly equal intensity, indicating that this surface is made up of roughly equal areas of $2 \times 1$ and $1 \times 2$ domains

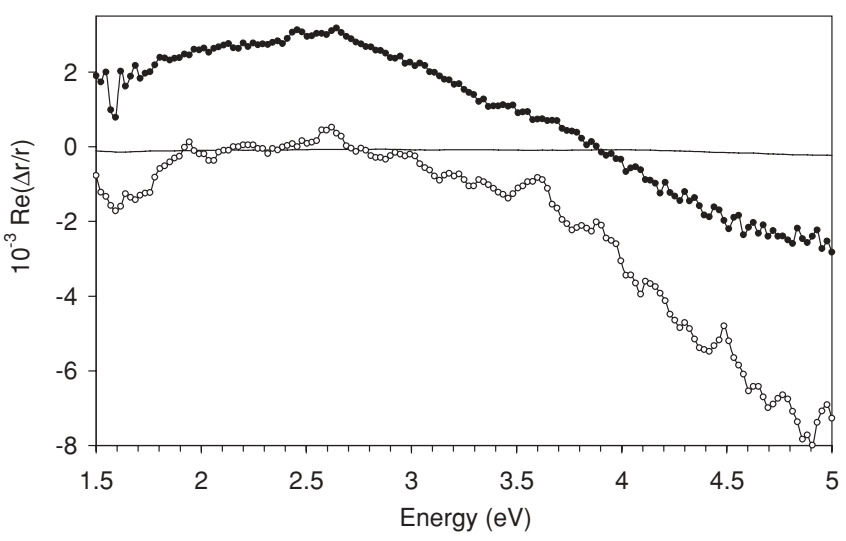

FIG. 2. RAS profiles for the vicinal $\mathrm{H} / \mathrm{C}(100)$ surfaces: $0^{\circ}$ (solid line), $2^{\circ}$ (open circles), and $4^{\circ}$ (filled circles). 


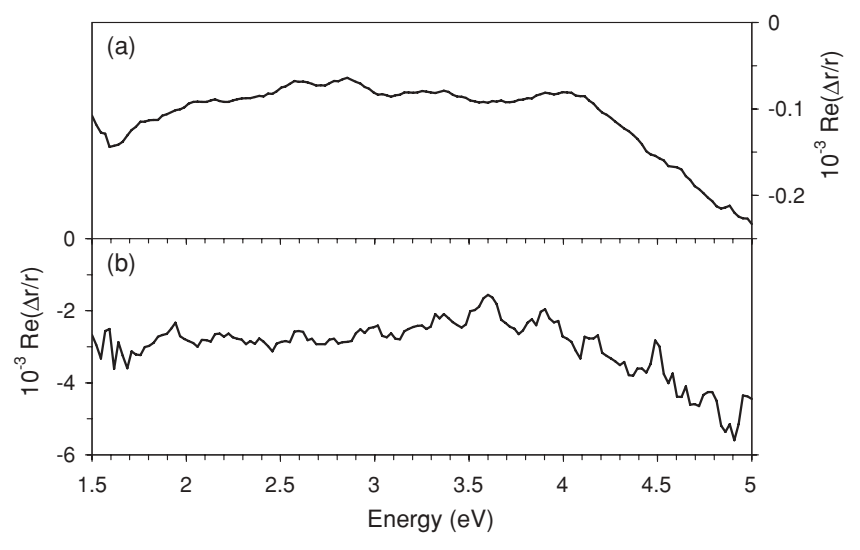

FIG. 3. RAS profile of (a) the flat $\mathrm{H} / \mathrm{C}(100) 0^{\circ}$ surface and (b) the difference RAS spectrum obtained from the subtraction of $\mathrm{H} / \mathrm{C}(100) 4^{\circ}$ from $\mathrm{H} / \mathrm{C}(100) 2^{\circ}$.

and, by implication, equal numbers of the associated steps. The steps will be of two types depending on the orientation of the dimerization direction of the reconstruction with respect to the direction of the step edge. Following $\mathrm{Chadi}^{28}$ we denote single height steps by $S_{A}$ and $S_{B}$, and double height steps by $D_{A}$ and $D_{B}$, the $\mathrm{A}$ and $\mathrm{B}$ subscripts denoting whether the dimerization directions on the upper terraces are orientated normal or parallel to the direction of the step edge, respectively. Chadi ${ }^{28}$ studied the energetics of steps on $\mathrm{Si}(100)$ surfaces vicinally oriented toward the [110] direction, the same geometry as in this work on diamond surfaces. He deduced energies for the formation of steps and found that the energy to form an $S_{A}$ step was an order of magnitude lower than that required to form an $S_{B}$ step, and that the energy to form a $D_{B}$ step was an order of magnitude lower than that required to form a $D_{A}$ step. A crucial finding was that energetically $D_{B}<S_{A}+S_{B}$, which explained why single-domain surfaces can be established by annealing vicinal $\mathrm{Si}(100)$ surfaces through the stabilization of double height steps and the elimination of single height steps. Single height steps are more common on nominally flat $\mathrm{Si}(100)$ surfaces, but $D_{B}$ steps become energetically more favorable with increasing vicinality through the elimination of $S_{A}$ and $S_{B}$ steps, which are forced to alternate on such surfaces.

It is clear that similar conditions pertain on vicinal diamond surfaces ${ }^{13,14}$ with the crucial difference that on hydrogen plasma-treated diamond (100) surfaces, like those studied here, STM results have revealed the presence of $D_{A}$ steps, and $D_{B}$ steps are absent. ${ }^{13}$

The possible equilibrium geometries of diamond (100) surfaces in the presence of single and double steps have been investigated. ${ }^{14}$ Interestingly, the equilibrium geometries previously proposed by Chadi for $\mathrm{Si}(100)^{28}$ may not be the most stable ones on the diamond (100) surface. The driving force for this difference is that diamond is stiffer and more likely to form double bonds than Si. This favors the formation of dimers with respect to the rebonding of the atoms at the step edges, thus changing the equilibrium geometry. We denote by $S_{A}, S_{B}, D_{A}$, and $D_{B}$ the geometries proposed by Chadi and by $S_{B}^{\prime}, D_{A}^{\prime}$, and $D_{B}^{\prime}$ the ones proposed by Tsai and Yeh. ${ }^{14}$ The models for the $S_{A}$ steps are the same for the two treatments. Another interesting point made by Tsai and $\mathrm{Yeh}^{14}$ concerns the role of hydrogen, where it is found that for hydrogen-free surfaces the $D_{B}^{\prime}$ step is the most stable, whereas when $\mathrm{H}$ is added to the $\mathrm{C}(100)$ surface the $D_{A}^{\prime}$ steps become energetically more favorable, in agreement with STM results by Kuang et al. ${ }^{13}$ which show only the presence of type A double steps. The STM images of Kuang et al. ${ }^{13}$ also show the presence of local $3 \times 1$ domains, which might occur when a row of atoms in the dihydride configuration "decorates" a single height step of type A.

As shown by previous ab initio calculations (see, for example, Ref. 43 and references therein), the dihydride phase is energetically unfavorable as compared to the monohydride phase, due to the steric hindrance between neighboring dihydride units. Nevertheless, stripes of dihydride regions may appear locally as a metastable phase.

It is also important to note that as found on $\mathrm{Si}(100)$ surfaces $S_{B}$ steps on vicinal $\mathrm{H} / \mathrm{C}(100)$ surfaces are ragged and accompanied by many kinks, while $S_{A}$ steps are smoother and have few kinks. ${ }^{13} \mathrm{~A}$ kink on an $S_{B}$ step requires the formation of an $S_{A}$ step at right angles to the step edge, while a kink on $S_{A}$ step requires the formation of an $S_{B}$ step at right angles to the step edge. The lower formation energy of $S_{A}$ steps means that kinks proliferate on $S_{B}$ steps but are rare on $S_{A}$ steps.

\section{B. Theoretical modeling of steps}

We have simulated the $S_{A}, S_{B}, S_{B}^{\prime}, D_{A}$, and $D_{A}^{\prime}$ steps following models proposed by Tsai and Yeh $^{14}$ and Chadi. ${ }^{28}$ In all cases the calculations were carried out with a DFT plane wave code ${ }^{44}$ simulating the surface with a slab of 12 layers of $\mathrm{C}$ saturated on the bottom layer with hydrogen. A $50 \mathrm{Ry}$ energy cutoff and LDA pseudopotentials were used. Particular care has been devoted to the choice of the vacuum region separating the replicas of the repeated slabs: We selected a vacuum region of about $20 \AA$. The top surface has been partially "cut" to describe upper and lower terraces separated by single and double steps, of type A and B. All dangling bonds have been saturated with hydrogen. The geometries used are shown in Figs. 4 and 5. Essentially, the Chadi and Tsai models differ in the number of $\mathrm{C}-\mathrm{C}$ dimers, which is larger in the Tsai model, and in the step edges, which are not decorated with $\mathrm{H}$ in the Chadi model. The calculated reflectance anisotropy is defined as

$$
\frac{\Delta R}{R}=\frac{\left(R_{y}-R_{x}\right)}{R},
$$

with $y$ along the dimer chains of the ideal C(100):H $2 \times 1$ surface. For the stepped surface, $y$ is always parallel to the step edge, and $x$ is perpendicular to it. $R_{x}$ and $R_{y}$ are calculated within the DFT-RPA approximation ${ }^{45}$ in terms of the slab polarizability as shown in Manghi et al. ${ }^{46}$

\section{Analysis of RAS}

As indicated below there are strong theoretical grounds for expecting that the RAS of a perfectly flat $\mathrm{H}$-terminated $\mathrm{C}(100)$ surface will be zero for energies below $4.5 \mathrm{eV}$. Combining this result with the experimental results we first carry out an empirical analysis of the experimental RAS results. We expect the nominally flat $\mathrm{H} / \mathrm{C}(100) 0^{\circ}$ surface to have very 


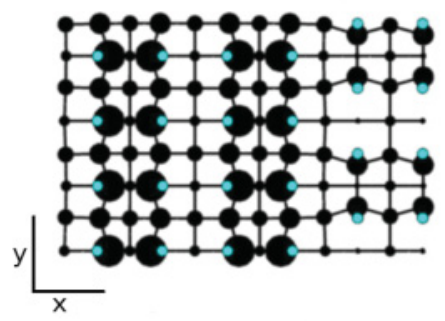

(a)
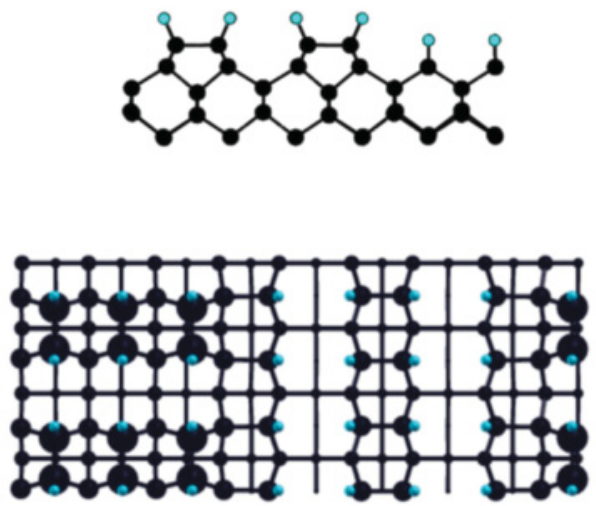

(b)
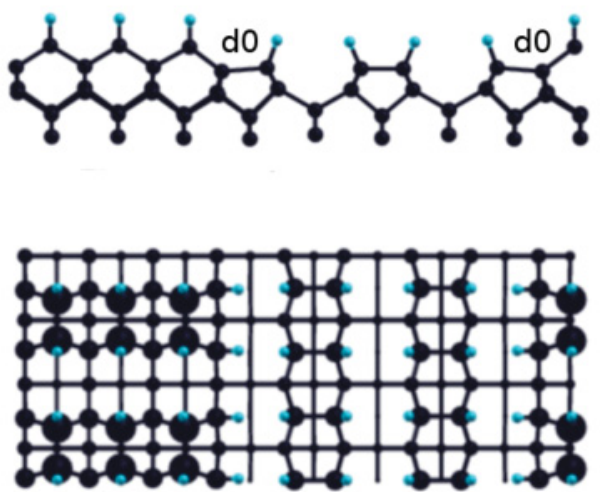

(c)

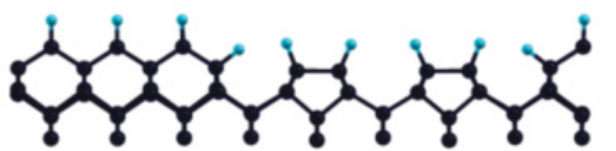

FIG. 4. (Color online) Geometries used to simulate single steps of type A (a), type B Chadi-like (b), and type B following Tsai and Yeh (c). In (b) the symbols d0 indicate very strained $\mathrm{C}-\mathrm{C}$ bonds $(1.64 \AA) .{ }^{49}$

few double height steps and approximately equal numbers of $S_{A}$ and $S_{B}$ single height steps. The latter expectation is confirmed by the LEED results, which showed that, as for the $\mathrm{H} / \mathrm{C}(100) 2^{\circ}$ surface, the $\mathrm{H} / \mathrm{C}(100) 0^{\circ}$ surface is composed of roughly equal areas of $1 \times 2$ and $2 \times 1$ domains. Figure 2 shows that the RAS observed from this surface is very small. However Fig. 3(a) shows the RAS of the $\mathrm{H} / \mathrm{C}(100) 0^{\circ}$ surface on a greatly expanded scale, and this shows that this surface does have a series of very small undulations in the RAS signal between 1.5 and $4 \mathrm{eV}$ and that there is a distinctive negative feature in the spectrum that increases linearly between 4 and $5 \mathrm{eV}$ and doubles in intensity over this energy range. We attribute this small RAS signal to the contributions from single

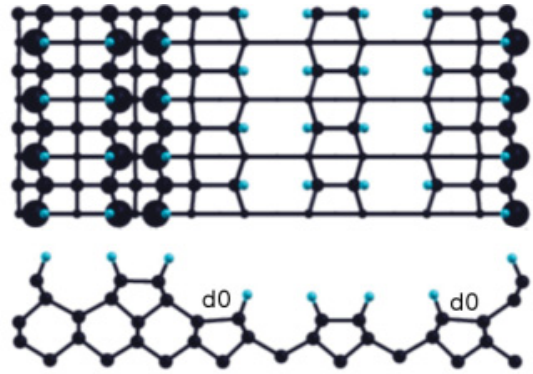

(a)

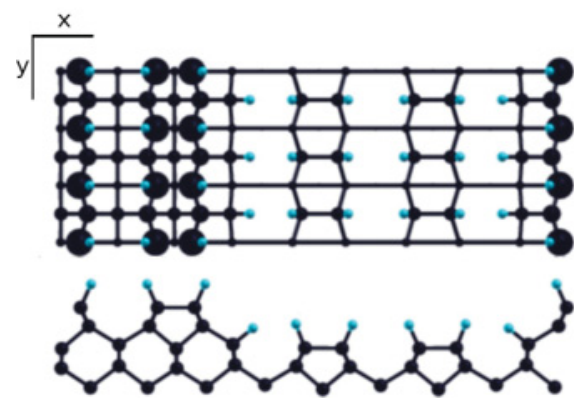

(b)

FIG. 5. (Color online) Models used to simulate double steps: (a) $D_{A}$ steps; (b) $D_{A}^{\prime}$ steps. The d0 in (a) indicates a very strained $\mathrm{C}-\mathrm{C}$ bond $(1.78 \AA){ }^{49}$

height steps $\left(S_{A}+S_{B}\right)$. Due to the lower formation energy of $S_{A}$ steps the $S_{B}$ steps will include many kinks, and the $S_{A}$ steps associated with kinks will be orientated at $90^{\circ}$ to the $S_{A}$ steps, leading to a preferential cancellation of the $S_{A}$ contribution to the RAS since $S_{A}$ steps will have far fewer kinks. Consequently the RAS signal of Fig. 3(a) should arise predominantly from $S_{B}$ steps.

The $\mathrm{H} / \mathrm{C}(100) 2^{\circ}$ and $4{ }^{\circ}$ surfaces give rise to much stronger RAS signals than the $\mathrm{H} / \mathrm{C}(100) 0^{\circ}$ surface Fig. 2 . The RAS signal from these two surfaces has essentially the same shape, though a different intensity, from 1.5 to $3.5 \mathrm{eV}$, and both show a linearly increasing negative signal, similar to that observed from the $\mathrm{H} / \mathrm{C}(100) 0^{\circ}$ surface, from 3.5 to $5 \mathrm{eV}$. The negative signal from 3.5 to $5 \mathrm{eV}$ is stronger on the $\mathrm{H} / \mathrm{C}(100) 2^{\circ}$ surface. The RAS results of Fig. 2 indicate that there is an imbalance in the contribution from steps that varies with the size of the vicinal angle. Since double height, $D_{A}$, steps are favored with increasing vicinality, we expect the $\mathrm{H} / \mathrm{C}(100) 2^{\circ}$ surface to have fewer $D_{A}$ steps but more $S_{A}$ and $S_{B}$ steps than the $\mathrm{H} / \mathrm{C}(100) 4^{\circ}$ surface. Furthermore while we expect more $S$ steps on the $\mathrm{H} / \mathrm{C}(100) 2^{\circ}$ surface than on the $\mathrm{H} / \mathrm{C}(100) 4^{\circ}$ surface, we expect the constraints of vicinality to ensure that on each surface the number of $S_{A}$ steps will be almost equal to the number of $S_{B}$ steps and that in each case the effect of kinks will be to lead to a preferential partial cancellation of the RAS contribution from $S_{A}$ steps. The general form of the RAS spectra in Fig. 2 may be explained if we assume that $D_{A}$ steps make a small, roughly flat but with a slight positively curved contribution to the RAS signal between 1.5 and $4 \mathrm{eV}$ and that the contribution from $\left(S_{A}+S_{B}\right)$ steps has the spectral shape shown in Fig. 3(a).

The expectation that there should be very few $D_{A}$ steps on the $\mathrm{H} / \mathrm{C}(100) 0^{\circ}$ surface accounts for the very small and essentially flat RAS observed between 1.5 to $4 \mathrm{eV}$ from this surface with the linearly decreasing RAS observed [Fig. 3(a)] 
TABLE I. Calculated minimum direct gap between surface states for the ideal C(100) $2 \times 1$ surface, clean and hydrogenated.

\begin{tabular}{lcc}
\hline \hline & $\begin{array}{c}\mathrm{C}(100) 2 \times 1 \\
(\mathrm{eV})\end{array}$ & $\begin{array}{c}\mathrm{C}(100): \mathrm{H} 2 \times 1 \\
(\mathrm{eV})\end{array}$ \\
\hline DFT & 2.2 & 3.1 \\
GW & 4.0 & 4.5 \\
\hline \hline
\end{tabular}

from $4 \mathrm{eV}$ onward arising from the $\left(S_{A}+S_{B}\right)$ contribution, as indicated earlier. The similarity in shape of the RAS observed in the range 1.5 to $4 \mathrm{eV}$ from the $\mathrm{H} / \mathrm{C}(100) 2^{\circ}$ and $4^{\circ}$ surfaces (Fig. 2) would then arise from both surfaces having a significant number of $D_{A}$ steps, with the stronger and positive signal from the $4{ }^{\circ}$ surface being caused by the greater number of $D_{A}$ steps on the more vicinal surface. The more negative RAS signal observed from the $\mathrm{H} / \mathrm{C}(100) 2^{\circ}$ surface from $3.5 \mathrm{eV}$ onward would arise from this surface having a greater contribution from $S$ steps, and hence a stronger $\left(S_{A}+S_{B}\right)$ RAS signal, than the $\mathrm{H} / \mathrm{C}(100) 4^{\circ}$ surface. These arguments suggest that the difference between RAS of the $\mathrm{H} / \mathrm{C}(100)$ $2^{\circ}$ and the $\mathrm{H} / \mathrm{C}(100) 4^{\circ}$ surfaces [Fig. 3(b)] should yield a spectral profile very similar to that observed from $\mathrm{H} / \mathrm{C}(100)$ $0^{\circ}$ surfaces. The result of this subtraction is shown in Fig. 3(b), and although noisy it does produce a similar profile to Fig. 3(a). The difference in absolute intensities between the two RAS spectra of Fig. 3 is attributable in the range $1.5-4 \mathrm{eV}$ to the spectrum of Fig. 3(a) having a negligible contribution from $D_{A}$ steps and in the range from $4 \mathrm{eV}$ onward and to an increased $\left(S_{A}+S_{B}\right)$ contribution from the difference of the RAS of the vicinal surfaces.

We now compare the results of the empirical analysis with the results of our theoretical calculations. The geometries used in our calculations to simulate the various step types are shown in Figs. 4 and $5 .^{47}$ Previous theoretical studies ${ }^{48-52}$ show that, as a result of the removal of surface states by the hydrogen termination, the electronic structure of the ideally flat H-terminated $\mathrm{C}(100)$ surface ensures that that there will be no contribution to the RAS of the $\mathrm{H} / \mathrm{C}(100)$ terraces below $4.5 \mathrm{eV}$. This is also confirmed by our ab initio calculations for the surface electronic gap (Table I) and for the RAS [Fig. 6(a)] since, in agreement with previous calculations, we find that the adsorption of hydrogen causes the disappearance of the $\pi$ and $\pi^{*}$ states from the forbidden gap region. We find a GW gap between surface states of about $4.5 \mathrm{eV}$ and a practically zero RAS in the energy range considered. Consequently any contribution to the RAS of $\mathrm{H}$-terminated diamond surfaces below this energy is expected to arise from optical transitions associated with steps and defects.

The results of the DFT theoretical calculations are shown in Fig. 6. First, the results for the monohydride $\mathrm{H} / \mathrm{C}(100) 0^{\circ}$ surface are in very good agreement with experiment, showing an essentially zero signal to $5 \mathrm{eV}$. The dihydride surface, on the other hand, gives rise to a negative signal above $2 \mathrm{eV}$. The absence of such a signal in the experimental spectrum of the flat surface is consistent with the expected absence of this phase in these experimental conditions. ${ }^{43}$ One surprising result is that the main feature of the experimental spectra, the negative signal from 3.5 to $5 \mathrm{eV}$, is reproduced only by the calculations for Chadi's models ${ }^{28}$ for the $S_{B}$ and $D_{A}$ steps.

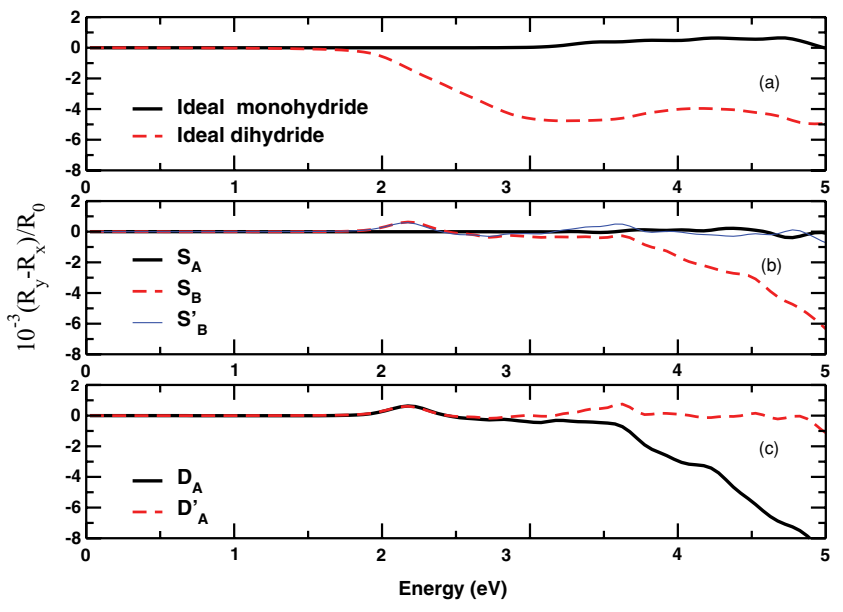

FIG. 6. (Color online) DFT-simulated RAS for (a) ideal flat $2 \times 1$ $\mathrm{C}(100): \mathrm{H}$ and $1 \times 1 \mathrm{C}(100): 2 \mathrm{H}$, (b) single steps, and (c) double steps. The $y$ direction is along the dimer rows in (a) and along the steps edge in (b) and (c).

The calculations for the model of the $S_{A}$ step and for the $S_{B}^{\prime}$ and $D_{A}^{\prime}$ step structures proposed by Tsai and $Y h^{14}$ all fail to yield the negative signal from $3.5 \mathrm{eV}$ onward, though they do reproduce the weak positive undulations that are seen at 2.5, 3.5 , and $4.5 \mathrm{eV}$ in the results for the $S_{B}$ and $D_{A}$ steps and in the experimental results for the $\mathrm{H} / \mathrm{C}(100) 2^{\circ}$ surface (Fig. 2). The comparison between theory and experiment suggests that although they are energetically less favorable than the step structures proposed by Tsai and Yeh, ${ }^{14} S_{B}$ and $D_{A}$ steps of the Chadi type are present on this surface. The fact that the RAS of $S_{A}$ steps is predicted to be zero does, of course, support the argument developed above that the contribution to the RAS from single height steps will be dominated by the contribution from $S_{B}$ steps but for a different reason. The close agreement of the RAS line shapes calculated for the $S_{B}$ and $D_{A}$ steps makes it impossible to analyze further the difference in step distribution on the $\mathrm{H} / \mathrm{C}(100) 2^{\circ}$ and $\mathrm{H} / \mathrm{C}(100) 4^{\circ}$ vicinal surfaces. The only significant disagreement between theory and experiment is the conclusion from the empirical comparison of results that the double height steps produce a broad positive contribution between 1.5 and $4 \mathrm{eV}$ that reaches a maximum value of $0.2 \times 10^{-3}$. The theoretical results are quite clear that all the step structures result in an essentially zero signal in this range. It is quite possible that this disagreement between theory and experiment could arise from the theoretical difficulty of modeling real steps and for the experiments in accurately determining the zero in the experimental results, though this would not account for the broad positively curved line shape.

\section{CONCLUSIONS}

We have investigated the surface geometry and the optical properties of flat and stepped hydrogen-terminated C(100) surfaces. The comparison between experimental RAS and theoretical simulations for the flat and vicinal surfaces suggests that, in the energy range studied here $(1-5 \mathrm{eV})$, the optical signal arises from surface steps.

The nominally flat $\mathrm{H} / \mathrm{C}(100) 0^{\circ}$ surface shows a small negative RAS signal that increases linearly and doubles in 
intensity between 4 and $5 \mathrm{eV}$. We attribute this linearly increasing negative signal between 4 and $5 \mathrm{eV}$ to the contribution from single height steps of the $S_{B}$ type proposed by Chadi. ${ }^{28}$ The RAS of the H/C(100) $2^{\circ}$ and $\mathrm{H} / \mathrm{C}(100) 4^{\circ}$ vicinal surfaces show stronger linearly increasing negative signals between 4 and $5 \mathrm{eV}$, and the calculations of both $S_{B}$ and $D_{A}$ double height steps of the Chadi type reproduce this line shape. However, the similarity in these line shapes prevents an analysis of the separate contributions of these steps to the RAS response of the vicinal surfaces. Further experimental work is desirable to quantify the parameters of step type and step density on flat and vicinal diamond surfaces; however, the number of experimental probes that are capable of distinguishing between step types on natural diamond surfaces is limited. While STM imaging of natural diamond surfaces generally provides insufficient resolution, high-resolution AFM imaging may offer a way forward.
Experimental and theoretical RAS results in combination with complementary high-resolution imaging would prove a powerful combination allowing further insight into the diamond surface and the RAS response and should facilitate the development of RAS as an in situ monitor of CVD diamond growth.

\section{ACKNOWLEDGMENTS}

MS and PW would like to acknowledge the De Beers Diamond Research Committee for the award of a $\mathrm{PhD}$ studentship. OP and MM acknowledge CPU time granted on CINECA, ENEA-CRESCO, and CASPUR supercomputer centers. The research leading to these results has received funding from the European Community's Seventh Framework Programme (FP7/2007-2013) under grant agreement number 211956 (ETSF user project no. 306).
${ }^{1}$ F. J. Himpsel, J. A. Knapp, J. A. VanVechten, and D. E. Eastman, Phys. Rev. B 20, 624 (1979).

${ }^{2}$ J. Ristein, Appl. Phys. A 82, 377 (2006).

${ }^{3}$ M. I. Landstrass and K. V. Ravi, Appl. Phys. Lett. 55, 975 (1989).

${ }^{4}$ F. Maier, M. Riedel, B. Mantel, J. Ristein, and L. Ley, Phys. Rev. Lett. 85, 3472 (2000).

${ }^{5}$ B. V. Spitsyn, L. L. Bouilov, and B. V. Derjaguin, J. Cryst. Growth 52, 219 (1981).

${ }^{6}$ B. Humbert, N. Hellala, J. J. Ehrhardt, S. Barret, and E. BauerGrosse, Appl. Surf. Sci. 254, 6400 (2008).

${ }^{7}$ K. Tsugawa et al., Diam. Relat. Mater. 8, 927 (1999).

${ }^{8}$ M. Brezeanu et al., Diam. Relat. Mater. 14, 499 (2005).

${ }^{9}$ R. E. Stallcup, A. F. Aviles, and J. M. Perez, Appl. Phys. Lett. 66, 2331 (1995).

${ }^{10}$ R. E. Stallcup and J. M. Perez, Phys. Rev. Lett. 86, 3368 (2001).

${ }^{11}$ R. E. Stallcup and J. M. Perez, Appl. Phys. Lett. 81, 4538 (2002).

${ }^{12}$ R. E. Stallcup, Y. Mo, T. W. Scharf, and J. M. Perez, Diam. Relat. Mater. 16, 1727 (2007).

${ }^{13}$ Y. Kuang, Y. Wang, N. Lee, A. Badzian, T. Badzian, and T. T. Tsong, Appl. Phys. Lett. 67, 3721 (1995).

${ }^{14}$ M. H. Tsai and Y. Y. Yeh, Phys. Rev. B 58, 2157 (1998).

${ }^{15}$ P. Weightman, D. S. Martin, R. J. Cole, and T. Farrell, Rep. Prog. Phys. 68, 1251 (2005).

${ }^{16}$ D. S. Martin and P. Weightman, Surf. Interface Anal. 31, 915 (2001).

${ }^{17}$ J. P. Harbison, D. E. Aspnes, A. A. Studna, L. T. Florez, and M. K. Kelly, Appl. Phys. Lett. 52, 2046 (1988).

${ }^{18}$ W. Richter and J. T. Zettler, Appl. Surf. Sci. 100/101, 465 (1996).

${ }^{19}$ T. Yasuda, L. Mantese, U. Rossow, and D. E. Aspnes, Phys. Rev. Lett. 74, 3431 (1995).

${ }^{20}$ T. Farrell, D. Hill, T. B. Joyce, T. J. Bullough, and P. Weightman, J. Cryst. Growth 175/176, 1217 (1997).

${ }^{21}$ R. J. Cole, S. Tanaka, P. Gerber, J. R. Power, T. Farrell, and P. Weightman, Phys. Rev. B 54, 13444 (1996).

${ }^{22}$ J. R. Power, P. Weightman, S. Bose, A. I. Shkrebtii, and R. Del Sole, Phys. Rev. Lett. 80, 3133 (1998).

${ }^{23}$ L. Kipp, D. K. Biegelsen, J. E. Northrup, L. E. Swartz, and R. D. Bringans, Phys. Rev. Lett. 76, 2810 (1996).
${ }^{24}$ R. J. Cole, J. A. Evans, P. Weightman, J. A. D. Matthew, D. A Woolf, and D. I. Westwood, Phys. Rev. B 49, 7528 (1994).

${ }^{25}$ J. A. Evans, A. D. Laine, P. Weightman, J. A. D. Matthew, D. A. Woolf, D. I. Westwood, and R. H. Williams, Phys. Rev. B 46, 1513 (1992).

${ }^{26}$ M. Palummo, O. Pulci, R. Del Sole, A. Marini, M. Schwitters, S. R. Haines, K. H. Williams, D. S. Martin, P. Weightman, and J. E. Butler, Phys. Rev. Lett. 94, 087404 (2005).

${ }^{27}$ M. Schwitters, D. S. Martin, P. Unsworth, T. Farrell, J. E. Butler, and P. Weightman, J. Phys. Condens. Matter 21, 364218 (2009).

${ }^{28}$ D. J. Chadi, Phys. Rev. Lett. 59, 1691 (1987).

${ }^{29}$ J. R. Power, T. Farrell, P. Gerber, S. Chandola, P. Weightman, and J. F. McGilp, Appl. Phys. Lett. 69, 176 (1996).

${ }^{30}$ M. Palummo, G. Onida, R. Del Sole, and B. S. Mendoza, Phys. Rev. B 60, 2522 (1999).

${ }^{31}$ C. E. Aumann, J. J. de Miguel, R. Kariotis, and M. G. Legally, Surf. Sci. 275, 1 (1992).

${ }^{32}$ J. D. O'Mahony, J. F. McGilp, F. M. Leibsle, P. Weightman, and C. F. J. Flipse, Semicond. Sci. Technol. 8, 495 (1993).

${ }^{33}$ R. Shioda and J. van der Weide, Phys. Rev. B 57, 6823 (1998).

${ }^{34}$ S. G. Jaloviar, J. L. Lin, F. Liu, V. Zielasek, L. McCaughan, and M. G. Lagally, Phys. Rev. Lett. 82, 791 (1999).

${ }^{35}$ W. G. Schmidt, F. Bechstedt, and J. Bernholc, Phys. Rev. B 63, 045322 (2001)

${ }^{36}$ P. Hohenberg and W. Kohn, Phys. Rev. 136, B864 (1964); W. Kohn and L. J. Sham, ibid. 140, A1113 (1965).

${ }^{37}$ L. Hedin and B. J. Lundquist in Solid State Physics, edited by H. Ehrereich, F. Seitz, and D. Turnbull (Academic Press, New York, 1969), Vol. 23, p. 1.

${ }^{38}$ B. D. Thoms, M. S. Owens, J. E. Butler, and C. Spiro, Appl. Phys. Lett. 65, 2957 (1994).

${ }^{39}$ P. Weightman, Phys. Scr. T 41, 277 (1992).

${ }^{40}$ J. Wei and J. T. Yates, Crit. Rev. Surf. Chem. 5, 1 (1995).

${ }^{41}$ S. R. Haines, K. H. Williams, N. Almond, M. Schwitters, D. S. Martin, J. E. Butler, and P. Weightman, J. Electron Spectroscop. 152, 33 (2006).

${ }^{42}$ C. I. Smith, A. Bowfield, N. J. Almond, C. P. Mansley, J. H. Convery, and P. Weightman J. Phys.: Condens. Matter 22, 392001 (2010). 
${ }^{43}$ J. A. Steckel, G. Kresse, and J. Hafner, Phys. Rev. B 66, 155406 (2002).

${ }^{44}$ P. Giannozzi et al., J. Phys. Condens. Matter 21, 395502 (2009).

${ }^{45}$ The RAS for the surfaces under consideration have been calculated within DFT using a mesh of $32 \times 32 \times 1 \mathrm{k}$ points in the Brillouin zone for the monohydride phase, $64 \times 64 \times 1 \mathrm{k}$ for the dihydride, $6 \times 12 \times 1 \mathrm{k}$ for $S_{A}$, and $8 \times 36 \times 1 \mathrm{k}$ points for the other surfaces.

${ }^{46}$ F. Manghi, R. Del Sole, A. Selloni, and E. Molinari, Phys. Rev. B 41, 9935 (1990).
${ }^{47}$ Images created with Xcrysden: A. Kokalj, Comput. Mater. Sci. 28, 155 (2003). Code available from [http://www.xcrysden.org/].

${ }^{48}$ V. I. Gavrilenko and A. I. Shkrebtii, Surf. Sci. 324, 226 (1995).

${ }^{49}$ V. I. Gavrilenko and F. Bechstedt, Phys. Rev. B 56, 3903 (1997).

${ }^{50}$ J. Furthmuller, J. Hafner, and G. Kresse, Phys. Rev. B 53, 7334 (1996).

${ }^{51}$ Z. Zhang, M. Wensell, and J. Bernholc, Phys. Rev. B 51, 5291 (1995).

${ }^{52}$ M. Marsili and O. Pulci, J. Phys. D: Appl. Phys. 43, 374016 (2010). 